ARTÍCULO DE INVESTIGACIÓN

Revista Facultad de Salud - RFS - Enero - Junio 2012;4-1:39-49

\title{
FACTORES ASOCIADOS A LA INASISTENCIA AL PRIMER TRIMESTRE DEL CONTROL PRENATAL EN MUJERES GESTANTES DE LA ESE SAN SEBASTIÁN DE LA PLATA, HUILA, 2009 Factors associated with first trimester period absence from prenatal control in pregnant women from ese san sebastian in La Plata, Huila, 2009
}

\author{
Ramiro Córdoba, Lina Paola Escobar, Lyda Lucero Guzmán*
}

Recibido: 2 de febrero de 2012 - Aceptado: 26 de junio de 2012

\section{Resumen}

Es un estudio analítico de casos y controles basado en la revisión de historias clinicas de las gestantes del programa de control prenatal que asistieron antes y después del primer trimestre de gestación en el periodo comprendido entre septiembre-diciembre de 2009 en la ESE San Sebastián de la Plata - Huila.

El objetivo es determinar los factores asociados con la inasistencia al control prenatal durante el primer trimestre. Se estudiaron 139 casos de gestantes con inicio tardio de los controles prenatales y 149 controles. Se realizó el análisis univariado y bivariado mediante el paquete estadístico SPSS versión 19 para Windows. Para estimar el riesgo de inicio tardio de los controles prenatales asociado con los factores de riesgo se calculó el Odds Ratio (OR), y se calcularon intervalos de confianza (IC) al 95\%. En los resultados, las edades de las 288 gestantes que acudieron a control prenatal oscilaron entre los 14 y 45 años.

El $77,1 \%$ de las gestantes pertenecían al régimen subsidiado. Nivel educativo superior a primaria es un factor protector, estadísticamente significativo para quienes lograron terminar la secundaria $(O R=1,85$, I.C $95 \%=1,05-3,14 p=0,015)$. El riesgo de inicio tardío del control prenatal se incrementó en la medida en que lo hacía el número de emba-

* Departamento de Salud Pública. Especialización en Epidemiología, Universidad Surcolombiana

Correspondencia: lydalguzman@mail.com razos, siendo estadisticamente significativo el antecedente de 4 a 6 gestaciones (I.C 95\%: 1,15-5,52; p-valor: 0,011). En conclusión, los principales factores asociados con el inicio del control prenatal después del primer trimestre son embarazo en adolescente, bajo nivel educativo; mujeres con 4 a 6 embarazos previos.

Palabras clave: control prenatal, estudio de casos y controles, gestante, factores de riesgo. 


\section{Abstract}

It is an analytical study of cases and controls based on medical records review of pregnant women from the prenatal program who attended before and after the first trimester of pregnancy from September to December 2009 at San Sebastian Hospital in La Plata - Huila.

This research aim is to determine the factors associated with no prenatal care during the first trimester period. It was studied a group of 139 cases of pregnant women with late onset of prenatal care and 149 controls. Univariate and bivariate analysis was made using SPSS version 19 for Windows. To estimate the risk of prenatal control late onset associated with risk factors, the odds ratio (OR) was calculated, and confidence intervals (CI) were calculated at 95\%. In the results, the ages of the 288 pregnant women attending antenatal care ranged between 14 and 45 .

$77.1 \%$ of pregnant women received the government's benefit. Educational level higher than primary education is a protective factor, statistically significant for those who managed to finish high school. $((O R=1,85 C I 95 \%=1,05-3,14 p=0,015)$. The risk of late onset of antenatal care increased as the number of pregnancies was higher, being the history of 4-6 pregnancies statistically significant (CI 95\%: 1, 155, 52; p-value: 0,011). In conclusion, the main factors associated with onset of prenatal care after the first trimester period are teen pregnancy, low educational levels, and women with 4-6 previous pregnancies.

Key words: prenatal care, case-control study, pregnant woman, risk factors

\section{INTRODUCCIÓN}

La mortalidad materna es un grave problema de salud pública que afecta a la mayoría de países pobres del mundo y tiene un gran impacto familiar, social y económico. Según la OMS en su informe sobre mortalidad materna publicado en el 2004, estimó que en el 2000 murieron 529.000 mujeres por complicaciones en el embarazo, en el parto y el puerperio. Menos del 1\% de esas muertes ocurrieron en países desarrollados, lo que indica que estas muertes pueden ser evitadas si se dispone de recursos y servicios de salud adecuados ${ }^{(1)}$.

El control prenatal es un sistema de consulta que evalúa la progresión normal del embarazo y descubre precozmente a través de la clínica, el laboratorio y la ecografía la aparición de problemas maternos y/o fetales que podrían presentarse; éste es considerado como una de las medidas más importantes para preservar la salud del binomio madre e hijo y está demostrado que contribuye a descender la mortalidad materna y perinatal ${ }^{(2)}$.

En Colombia se estableció como norma para la atención de gestantes la captación temprana después de la primera falla menstrual y antes de la semana 12 de gestación, con consultas mensuales de seguimiento por médico o enfermera hasta la semana 36 y luego cada 15 días hasta el parto ${ }^{(2)}$. El Plan Nacional de Salud Pública para el cuatrienio 2007-2010, incluye como una prioridad la salud sexual y reproductiva, dentro de ella se cita la importancia de mejorar la cobertura y calidad de la atención en las gestantes, implementar mecanismos que permitan su captación temprana, identificar y tratar riesgos biológicos y psicosociales e implementar mecanismos para facilitar el acceso a los servicios de salud de las embarazadas de zonas rurales y de conflicto armado con el fin de disminuir la tasa de mortalidad materna ${ }^{(3)}$. En el departamento del Huila, en el munici- 
pio de la Plata en el año 2008 se atendieron a 816 mujeres gestantes, de las cuales el $55,14 \%$ no asistieron a los controles del primer trimestre de embarazo ${ }^{(4)}$. La investigación realizada tiene como objetivo identificar las características socio demográficas (edad, seguridad social, nivel educativo, estado civil) de las embarazadas adscritas al control prenatal en la ESE San Sebastián de la Plata Huila en el año 2009 y establecer una caracterización de los factores asociados con la asistencia al control prenatal, desde el primer trimestre de embarazo en comparación con la asistencia a partir de la semana 14 de embarazo.

\section{MATERIALES Y MÉTODOS}

Se realizó un estudio analítico de tipo casos y controles con los registros clínicos de las gestantes inscritas al control prenatal en la ESE San Sebastián de la Plata-Huila, que asistieron antes y después de la semana 14 de gestación en el periodo comprendido entre septiembre-diciembre del 2009. La población del estudio fue el total de gestantes inscritas al control prenatal. Se aplicó una lista de chequeo a las historias clínicas, determinando 139 casos y 149 controles. La información se registró directamente en una base de datos Excel 2007. Se realizó el análisis univariado y bivariado mediante el paquete estadístico SPSS versión 19 para Windows. Con el fin de estimar el riesgo de inicio tardío a los controles prenatales asociado con los diferentes factores de riesgo se calculó la razón de productos cruzados u Odds Ratio (OR) como aproximación del riesgo relativo (estadísticamente significativo OR $>1$ ) e intervalos de confianza (IC) al 95\% y PValor $(<0,05)$. Para controlar los efectos de los potenciales factores confusores se utilizó la estratificación y el procedimiento de MantelHaenszel. Se tabularon los datos y se realizó un análisis de medidas de tendencia central y de dispersión (media, mediana, desviación estándar), calculando la diferencia entre los grupos con la prueba $t$ de student y para las variables categóricas se utilizó el chi cuadrado.

\section{RESUlTADOS}

Para la realización del presente estudio se incluyeron un total de 288 mujeres en estado de gestación inscritas al control prenatal en la ESE San Sebastián del Municipio de La Plata Huila, en el período comprendido entre septiembre y diciembre del 2009.

El análisis de los factores sociodemográficos reportó que las edades del total de gestantes que acudieron a control prenatal oscilaron entre los 14 y 45 años con una mediana de 23 (tabla 1). En lo referente al régimen de afiliación al sistema de salud, se estableció que la mayoría de las gestantes (77,1\%) pertenecían al Régimen Subsidiado, en tanto que las restantes $(22,9 \%)$ eran vinculadas. No se encontraron gestantes del régimen contributivo. En cuanto al nivel educativo de las gestantes, un $57,6 \%$ tiene como máximo la primaria completa, un 35,5\% han ingresado a nivel de secundaria, y solo el $1,7 \%$ ha ingresado a la universidad.Se encontró que el estado civil es en su mayoría era casadas $(43,7 \%)$. La distribución por área de residencia determinó que las tres cuartas partes de la población eran procedentes del área rural. Al analizar los factores ginecoobstétricos, la distribución por trimestre de inicio de control prenatal de las gestantes evidenció un mayor porcentaje $(51,7 \%)$ en las que ingresaron durante el primer trimestre de gestación, constituyendo el grupo de controles; mientras que el 48,3\% lo hicieron después del primer trimestre constituyendo el grupo de casos. Los antecedentes de embarazos previos oscilaron entre 0 y 1 con un promedio de 1,82 gestaciones $\pm 2,21$ y una mediana de 1 . Se observó un porcentaje importante de gestantes $(35,8 \%)$ que no tenían historia concepcional (tabla 2). 
Tabla 1. Características Socio demográficas de las gestantes que asistieron al primer control prenatal en la ESE San Sebastián del municipio de la Plata, Huila desde septiembre a diciembre del año 2009.

\begin{tabular}{|c|c|c|c|c|}
\hline Edad de la madre & No. Gestantes & $\begin{array}{c}\text { FA } \\
\text { Frecuencia } \\
\text { Acumulada }\end{array}$ & $\begin{array}{c}\text { Fr } \\
\text { Frecuencia } \\
\text { relativa }\end{array}$ & $\begin{array}{c}\text { FR } \\
\text { Frecuencia } \\
\text { Acumulada }\end{array}$ \\
\hline$<15$ & 7 & 7 & $2,4 \%$ & $2,4 \%$ \\
\hline 15 A 19 & 70 & 77 & $24,3 \%$ & $26,7 \%$ \\
\hline 20 A 24 & 102 & 179 & $35,4 \%$ & $62,2 \%$ \\
\hline 25 A 29 & 53 & 232 & $18,4 \%$ & $80,6 \%$ \\
\hline 30 A 34 & 26 & 258 & $9,0 \%$ & $89,6 \%$ \\
\hline 35 A 39 & 21 & 279 & $7,3 \%$ & $96,9 \%$ \\
\hline 40 A 44 & 8 & 287 & $2,8 \%$ & $99,7 \%$ \\
\hline 45 Y MAS & 1 & 288 & $0,3 \%$ & $100,0 \%$ \\
\hline$X=24,04 \pm 6,7$ & Me: 23 R: 14-45 & & & \\
\hline Régimen de afiliación al SGSSS & & FA & Fr & FR \\
\hline Contributivo & 0 & 0 & $0 \%$ & $0 \%$ \\
\hline Subsidiado & 222 & 222 & $77,1 \%$ & $77,1 \%$ \\
\hline Vinculado & 66 & 288 & $22,9 \%$ & $100,0 \%$ \\
\hline Escolaridad & & FA & Fr & FR \\
\hline Ninguna-analfabeta & 1 & 1 & $0,3 \%$ & $0,3 \%$ \\
\hline Primaria incompleta & 71 & 72 & $24,7 \%$ & $25,0 \%$ \\
\hline Primaria completa & 94 & 166 & $32,6 \%$ & $57,6 \%$ \\
\hline Secundaria incompleta & 52 & 218 & $18,1 \%$ & $75,7 \%$ \\
\hline Secundaria completa & 50 & 268 & $17,4 \%$ & $93,1 \%$ \\
\hline Universidad & 5 & 273 & $1,7 \%$ & $94,8 \%$ \\
\hline No registra & 15 & 288 & $5,2 \%$ & $100,0 \%$ \\
\hline Estado civil & & FA & Fr & FR \\
\hline Casada & 126 & 126 & $43,7 \%$ & $43,7 \%$ \\
\hline Soltera & 86 & 212 & $29,9 \%$ & $73,6 \%$ \\
\hline Unión libre & 76 & 288 & $26,4 \%$ & $100,0 \%$ \\
\hline Área & & FA & $\mathrm{Fr}$ & FR \\
\hline Urbano & 70 & 70 & $24,3 \%$ & $24,3 \%$ \\
\hline Rural & 218 & 288 & $75,7 \%$ & $100,0 \%$ \\
\hline
\end{tabular}


Tabla 2. Características ginecoobstétricas de las gestantes que asistieron al primer control prenatal en la ESE San Sebastián de la Plata, Huila desde septiembre a diciembre del año 2009

\begin{tabular}{lcccc}
\hline No. Gestantes & FA & $\begin{array}{c}\text { Fr } \\
\text { Frecuencia } \\
\text { Acumulada }\end{array}$ & $\begin{array}{c}\text { Frecuencia } \\
\text { Relativa }\end{array}$ & $\begin{array}{c}\text { Frecuencia } \\
\text { Absoluta }\end{array}$ \\
\hline Primestre & 149 & 149 & $51,7 \%$ & $51,7 \%$ \\
Segundo & 113 & 262 & $39,3 \%$ & $91,0 \%$ \\
Tercero & 26 & 288 & $9,0 \%$ & $100,0 \%$ \\
Total & 288 & & $100 \%$ & \\
\hline
\end{tabular}

Número de embarazos previos

\begin{tabular}{lcccc}
\hline 0 A 103 & 103 & $35,8 \%$ & $35,8 \%$ & \\
1 A 3 & 128 & 231 & $44,4 \%$ & $80,2 \%$ \\
4 A 6 & 46 & 277 & $16,0 \%$ & $96,2 \%$ \\
7 y mas & 11 & 288 & $3,8 \%$ & $100,0 \%$ \\
Total & 288 & & $100 \%$ & \\
$?=1,82 \mathrm{DE}= \pm 2,21 \mathrm{Me}=1 \mathrm{R}: 0-11$ & & & & \\
\hline
\end{tabular}

Número de hijos previos

\begin{tabular}{|c|c|c|c|c|}
\hline 0 A 112 & 112 & $38,9 \%$ & $38,9 \%$ & \\
\hline $1 \mathrm{~A} 3$ & 136 & 248 & $47,2 \%$ & $86,1 \%$ \\
\hline $4 \mathrm{~A} 6$ & 32 & 280 & $11,1 \%$ & $97,2 \%$ \\
\hline 7 y más & 8 & 288 & $2,8 \%$ & $100,0 \%$ \\
\hline Total & 288 & & $100 \%$ & \\
\hline \multicolumn{5}{|c|}{$?=1,57 \mathrm{DE}= \pm 1,97 \mathrm{Me}=1 \mathrm{R}=0-10$} \\
\hline \multicolumn{5}{|c|}{ Número de abortos previos } \\
\hline 0 A 245 & 245 & $85,1 \%$ & $85,1 \%$ & \\
\hline$>=1$ & 43 & 288 & $14,9 \%$ & $100,0 \%$ \\
\hline Total & 288 & & $100 \%$ & \\
\hline \multicolumn{5}{|c|}{$?=0,19 \mathrm{DE}= \pm 0,55 \mathrm{Me}=0 \mathrm{R}=0-4$} \\
\hline \multicolumn{5}{|c|}{ Número de mortinatos previos } \\
\hline 0 & 277 & 277 & $96,2 \%$ & $96,2 \%$ \\
\hline$>=1$ & 11 & 288 & $3,8 \%$ & $100,0 \%$ \\
\hline Total & 288 & & $100 \%$ & \\
\hline
\end{tabular}


El análisis bivariado permitió determinar diferencias en cuanto al factor edad entre los casos y controles. Se observó que $45 \%$ de los casos eran mujeres en los extremos de vida fértil vs $29 \%$ en los controles. Sin embargo al medir el OR esta diferencia fue estadísticamente significativa, solamente para el grupo menor de 20 años (OR 1,94, IC 1.09-3.26) (Tabla 3).Las gestantes que lograron tener estudios superiores a primaria tuvieron un aparente factor protector, el cual sólo fue estadísticamente significativo para quienes terminaron la secundaria $(\mathrm{OR}=0,39$ I.C 95\%=0,19-0,79). (Tabla 3).

El estado civil de las gestantes tuvo una distribución muy similar entre las que iniciaron tardíamente el control prenatal frente a las que lo hicieron en forma temprana. No obstante, aunque en las solteras y en las de unión libre se observaron riesgos mayores para el inicio tardío de los controles prenatales, estos no fueron estadísticamente significativos (solteras: OR=1,39; I.C:95\%: 0,77-2,51), (unión libre: $\mathrm{OR}=1,09$; I.C:95\%:0,59-2,01) (ver tabla 3). El riesgo de iniciar control prenatal en forma tardía fue 1,56 veces mayor en las mujeres del área rural frente a las del área urbana (OR=1,56; I.C:95\%: 0,87-2,7) (tabla 3).

$\mathrm{Al}$ analizar los riesgos relativos de los antecedentes ginecoobstétricos se observó que en la medida en que la historia de embarazos aumentaba, tomando como grupo de referencia la ausencia de estos, también lo hacía el OR, aunque solamente fue estadísticamente significativo en el intervalo de 4 a 6 gestaciones en quienes el riesgo de asistir tardíamente a los controles prenatales fue 2,5 veces mayor frente a las que lo hicieron en forma temprana (I.C 95\%: 1,15-5,52). Similar situación ocurrió en las gestantes que han tenido hijos previos, entre mayor era el número de hijos, el riesgo de asistir tardíamente al control prenatal aumentaba; aunque solamente fue estadísticamente significativo en los que tuvieron de 4 a 6 hijos, con un riesgo aumentado en 2,73 veces más (I.C 95\%: 1,10-6,5). El número de abortos y mortinatos previos incrementaron el riesgo 1,43 y 1,3 veces más en ese orden, pero no fue estadísticamente significativo (tabla 4). Los abortos y los mortinatos previos se presentaron hasta en tres oportunidades en los casos, en tanto que en los controles hubo una leve variación observándose antecedentes de hasta 4 abortos y 2 mortinatos como máximo. La comparación de las medias no arrojó significancia estadística para ninguno de los dos eventos (abortos: p-valor: 0,526; mortinatos: p-valor: 0,476) (tabla 5).

\section{DISCUSIÓN}

La asistencia de las gestantes al programa de detección temprana de alteraciones del embarazo se considera como una de las medidas más importantes para preservar la salud materno-fetal a nivel nacional e internacional. La asistencia al control prenatal permite conocer factores de riesgo, que al ser detectados y controlados oportunamente por el equipo de salud, disminuyen la morbimortalidad materno fetal $^{5}$. El presente estudio determina los factores de riesgo involucrados en la no asistencia al control prenatal en el primer trimestre de gestación en la ESE San Sebastián de la Plata Huila. En nuestro país existen pocos estudios epidemiológicos relacionados con este problema específico, pues la mayoría se centran en conocer los motivos por los cuales las gestantes dejan de asistir al control prenatal en forma generalizada. En esta investigación, al igual que en la reportada por Valadez et al. ${ }^{6}$, la población adolescente y el bajo nivel educativo son factores relacionados directamente con el inicio tardío de los controles prenatales. La oportunidad de educación en muchas comunidades es menor para las mujeres y en nuestra sociedad, el embarazo en la adolescente reduce en mayor escala esta oportunidad(7). El bajo nivel social y cultural hacen que las mujeres desconozcan sus derechos y son una desventaja para el acceso a la información y a los servicios de salud ${ }^{(8)}$. Otros autores demuestran 
Tabla 3. Modelo bivariado de los factores de riesgo socio demográficos asociados al inicio tardío de los Controles Prenatales en la ESE San Sebastián del municipio de la Plata, Huila en el periodo comprendido entre los meses de septiembre y diciembre de 2009.

Edad de la gestante al inicio del control prenatal

\begin{tabular}{|c|c|c|c|c|c|c|}
\hline & \multicolumn{2}{|c|}{ Casos } & \multicolumn{2}{|c|}{ Controles } & \multirow{2}{*}{ OR } & \multirow{2}{*}{ P-Valor } \\
\hline & No & $\%$ & No & $\%$ & & \\
\hline$<20$ años & 45 & 32 & 32 & 21 & 1,94 & 0,015 \\
\hline 20 a 34 años (1) & 76 & 55 & 105 & 70 & 1 & \\
\hline 35 y más años & 18 & 13 & 12 & 8 & 2,07 & 0,769 \\
\hline
\end{tabular}

Nivel educativo

\begin{tabular}{lcccccc}
\hline Analfabeta & 0 & 0 & 1 & 1 & 0 & 0,333 \\
Primaria incompleta & 39 & 28 & 32 & 21 & 1,43 & 0,195 \\
Primaria completa & 55 & 40 & 39 & 26 & 1,85 & 0,015 \\
Secundaria incompleta & 20 & 14 & 32 & 21 & 0,61 & 0,118 \\
Secundaria completa & 15 & 11 & 35 & 23 & $\mathbf{0 , 3 9}$ & 0,004 \\
Universidad & 1 & 1 & 4 & 3 & 0,26 & 0,201 \\
No registra & 9 & 6 & 6 & 4 & 1,65 & 0,35 \\
\hline
\end{tabular}

\section{Régimen de salud}

\begin{tabular}{lcccccc}
\hline Contributivo & 0 & $\%$ & 0 & $\%$ & 0 & \\
Subsidiado(1) $^{(1)}$ & 110 & 79 & 112 & 75 & 1 & \\
Vinculado & 29 & 21 & 37 & 25 & 0,79 & 0,414 \\
\hline
\end{tabular}

Estado civil de las gestantes que asistieron al control prenatal

\begin{tabular}{lllllll}
\hline Casada $^{(1)}$ & 57 & 41 & 69 & 46 & 1 & \\
Soltera & 46 & 33 & 40 & 27 & 1,39 & 0,239 \\
Unión libre & 36 & 26 & 40 & 27 & 1,09 & 0,769 \\
\hline
\end{tabular}

Área de residencia de la gestante

\begin{tabular}{lcccccc}
\hline Urbano $^{(1)}$ & 28 & $20 \%$ & 42 & $28 \%$ & 1 & \\
Rural & 111 & $80 \%$ & 107 & $72 \%$ & 1,56 & 0,111 \\
\hline
\end{tabular}

(1) Grupo de Referencia 
Tabla 4. Modelo Bivariado de los Factores de Riesgo Ginecoobstétricos asociados al inicio tardío de los Controles Prenatales en La ESE San Sebastián del municipio de la Plata, Huila en el periodo comprendido entre los meses de septiembre y diciembre de 2009

\begin{tabular}{|c|c|c|c|c|c|c|}
\hline \multicolumn{7}{|c|}{ Antecedentes ginecobstetricos } \\
\hline & \multicolumn{2}{|c|}{ Casos } & \multicolumn{2}{|c|}{ Controles } & \multirow{2}{*}{ OR } & \multirow{2}{*}{ P-Valor } \\
\hline & No & $\%$ & No & $\%$ & & \\
\hline \multicolumn{7}{|c|}{ Número de embarazos previos } \\
\hline Ninguno $^{(1)}$ & 44 & 31,7 & 59 & 39,6 & 1 & \\
\hline $1 \mathrm{~A} 3$ & 58 & 41,7 & 70 & 47,0 & 1,11 & 0,694 \\
\hline 4 A 6 & 30 & 21,6 & 16 & 10,7 & 2,51 & 0,011 \\
\hline 7 y más & 7 & 5,0 & 4 & 2,7 & 2,35 & 0,187 \\
\hline Total & 139 & 100 & 149 & 100 & & \\
\hline \multicolumn{7}{|c|}{ Número de hijos previos } \\
\hline Ninguno $^{(1)}$ & 50 & 36,0 & 62 & 41,6 & 1 & \\
\hline $1 \mathrm{~A} 3$ & 61 & 43,9 & 75 & 50,3 & 1,01 & 0,974 \\
\hline 4 A 6 & 22 & 15,8 & 10 & 6,7 & 2,73 & 0,017 \\
\hline 7 y más & 6 & 4,3 & 2 & 1,3 & 3,72 & 0,098 \\
\hline Total & 139 & 100 & 149 & 100 & & \\
\hline \multicolumn{7}{|c|}{ Número de abortos previos } \\
\hline Ninguno $^{(1)}$ & 115 & 83 & 130 & $87 \%$ & 1 & \\
\hline$>=1$ & 24 & 17 & 19 & $13 \%$ & 1,43 & 0,282 \\
\hline Total & 139 & 100 & 149 & $100 \%$ & & \\
\hline \multicolumn{7}{|c|}{ Numero de mortinatos previos } \\
\hline Ninguno $^{(1)}$ & 133 & 96 & 144 & 97 & & \\
\hline$>=1$ & 6 & 4 & 5 & 3 & 0,18 & 0,67 \\
\hline Total & 139 & 100 & 149 & 100 & & \\
\hline
\end{tabular}

asociación estadística entre el inicio tardío de los controles prenatales y el hecho de no tener servicios médicos de seguridad social, situación que generalmente se comporta como factor de riesgo ${ }^{(9,10)}$.
No se logró comprobar que el estado civil soltera estuviera asociado con el riesgo de inicio tardío de los controles prenatales, tal como si lo expresan otros autores, ${ }^{(11-14)}$ quienes encontraron que las mujeres con esta condición 
Tabla 5. Factores de riesgo ginecoobstétricos asociados al inicio tardío de los controles prenatales en la ESE San Sebastián del municipio de la Plata, Huila en el periodo comprendido entre los meses de septiembre y diciembre de 2009

\begin{tabular}{lcccccc}
\hline & Mínimo & Máximo & Mediana & Media & DE & P* \\
\hline Gestaciones previas & & & & & & \\
\hline Casos & 0 & 11 & 1 & 2,16 & 2,372 & 0,013 \\
Controles & 0 & 11 & 1 & 1,51 & 1,995 & \\
\hline Hijos vivos previos & & & & & \\
\hline Casos & 0 & 10 & 1 & 1,86 & 2,155 & 0,018 \\
Controles & 0 & 10 & 1 & 1,30 & 1,743 & \\
\hline Abortos previos & & & & & & \\
\hline Casos & 0 & 3 & 0 & 0,22 & 0,535 & 0,526 \\
Controles & 0 & 4 & 0 & 0,17 & 0,566 & \\
\hline Mortinatos previos & & & & & & \\
\hline Casos & 0 & 3 & 0 & 0,06 & 0,345 & 0,476 \\
Controles & 0 & 2 & 0 & 0,04 & 0,229 & \\
\hline
\end{tabular}

* $p$ de prueba $t$ para diferencia de medias

tienen mayor riesgo de tener un control prenatal incompleto. Sin embargo, para Quelopana del Valle ${ }^{(15)}$ ninguna de las variables sociodemográficas, incluyendo el estado civil, fueron predictores del inicio precoz de los controles prenatales.

A pesar de que algunos estudios han encontrado menor acceso al control prenatal en mujeres del área rural ${ }^{(16-19)}$ en este, al igual que en el realizado por Vecino ${ }^{20}$, no se encontró una asociación estadísticamente significativa; lo anterior puede obedecer a que la Empresa Social del Estado del primer nivel presta sus servicios en muchas de las veredas del municipio como consecuencia de las jornadas de salud realizadas en el área rural.

En cuanto a los antecedentes ginecoobstétricos, se encontró que al igual que Valadez et al. ${ }^{21}$, el riesgo de inicio tardío de los controles prenatales se incrementó en la medida que lo hacía el número de embarazos e hijos vivos, siendo estadísticamente significativo entre el 4 y 6 para ambos casos. Las mujeres que tengan 4 o más hijos presentan razones de mortalidad materna superiores a 120, mientras que las que tienen 2, 4 o menos hijos presentan razones por debajo de $64^{(22)}$. Por tanto, factores de riesgo como la multiparidad en rango de 4 a 6 hijos constituye un factor de riesgo para inasistencia temprana al control prenatal como se demostró en nuestro estudio y a su vez es un factor de riesgo conocido para mortalidad materna.

La situación de la mortalidad materna está condicionada por la implementación de políticas de salud pública eficaces, orientadas hacia la promoción de la maternidad segura, que se lograra partiendo desde la captación oportuna de las gestantes en cada institución de salud de nuestro país. 


\section{CONCLUSIONES}

Identificamos factores de riesgo sociodemográficos y ginecoobstétricos que se relacionan directamente con la ausencia de las gestantes al control médico en el primer trimestre de embarazo y por tanto, limitan su captación temprana en el programa de control prenatal de la institución. A su vez son factores que limitan la identificación precoz de problemas del embarazo, parto y puerperio, que conocidos y resueltos a tiempo producirían una disminución de la morbimortalidad materna y fetal en nuestra región.

Los principales factores de riesgo sociodemográficas asociados al inicio tardío de los controles prenatales en la ESE San Sebastián del municipio la Plata Huila en el periodo de tiempo estudiado fueron: ser gestante adolescente, estado civil soltera, bajo nivel educativo. El principal factor de riesgo ginecoobstétrico asociado al inicio tardío a los controles prenatales fue determinado por las mujeres que tenían entre cuatro a seis embarazos previos. Se encontró asociación estadística entre el inicio tardío de los controles y el hecho de no tener servicios médicos de seguridad social, condición que generalmente se comporta como factor de riesgo.

\section{RECOMENDACIONES}

Recomendamos realizar estudios poblacionales que permitan evaluar estrategias eficaces para mejorar la adherencia al control prenatal, con énfasis en la prevención de la enfermedad y en la disminución de la morbimortalidad maternofetal. Con las actividades de promoción de la salud y prevención de la enfermedad podemos identificar factores de riesgo que aumentan las posibilidades de complicaciones en el curso del embarazo, en el parto y en el puerperio. Por tanto, se debe lograr que las mujeres diferencien lo normal de lo anormal en este proceso, se identifiquen en el momento en que deben buscar la atención del personal de salud; así lograremos mejorar la calidad de la atención médica para el cuidado de la salud de la gestante.

Es importante apoyar el proceso de articulación de la ESE Municipal para realizar de manera efectiva acciones tendientes a captar a las gestantes en el primer trimestre de embarazo con los Equipos de Atención Primaria -APS- que desde el año 2005 vienen laborando en beneficio de la población materno-infantil del Departamento del Huila. Igualmente, debemos trabajar conjuntamente con las EPS, Dirección Local de Salud DLS-, líderes comunitarios, y Familias en Acción, diferentes redes comunitarias e institucionales y demás sectores del Sistema General de Seguridad Social para que apoyen este proceso.

\section{REFERENCIAS}

1 World Health Organization. Maternal Mortality in 2000: estimates developer (monografía en internet). Geneva: WHO, UNICEF and UNFPA: 2004 (citado 2005 mar 15) Disponibleen:http:/ / www.who.int/reproductive;health / publications/maternal_mortality_2000/ mme.pdf.

2 Ministerio de la Protección Social. República de Colombia. Resolución 412 de 2000.

3 Ministerio de la Protección Social. República de Colombia. Plan Nacional de Salud Pública, 2007-2010.

4 Boletín salud sexual y Reproductiva. Departamento del Huila, 2008.

5 OMS-OPS. La salud en las Américas. Maternidad sin riesgo. Edición de 2002, volumen 1.

6 Belizán, José María, Villar, José, Belizán, María M, Garrote, Nora. Asistencia de embarazadas a la consulta prenatal en maternidades públicas de Rosario, Argentina. Bol. Of.sanit. Panam, 1979;2:121-130.

7 Camacho A, OPS, Perfil de salud sexual y reproductiva de los y las adolescentes y jóvenes de América Latina y el Caribe revisión bibliografía 1988-1998:23-25, 60-64. Información ubicada en http://www.adolec. org/pdf/ Perfilweb.pdf. 
8 Organización Panamericana de la Salud. Reducción de la mortalidad y la morbilidad materna: Consenso Estratégico Interagencial para América Latina y el Caribe. Washington D.C: OPS, 2003.

9 Cáceres M, Flor de María. El control prenatal: Una reflexión urgente. En: Revista Colombiana de Obstetricia y Ginecología, volumen 60, número 2. Bogotá, abril/junio 2009:4.

10 Cáceres M, Flor de María, et al. La mortalidad materna en el Hospital Universitario Ramón González Valencia entre 1994-2004. En: Revista Colombiana de Obstetricia y Ginecología, volumen 58, número 2. Bogotá. 2007.

11 Hans Albrecht, Von Wandenfels, Maryann Pflans, Ramón Jorge. Factores que influyen en la concurrencia al control prenatal en Montevideo, Uruguay. En: Revista Médica de Uruguay, volumen 8, número 1. Uruguay. 1992

12 Belizán JM, Conde-Agudelo A, Lammers C. Maternal-perinatal morbidity and mortality associated with adolescent pregnancy in Latin America: Cross-sectional study. American Journal of Obstetrics and Gynecology. 2005;192:342-9.

13 Johnson S, Gibbs E, Kogan M, Knapp C, Hansen $\mathrm{JH}$. Massachusetts prenatal care survey factors related to prenatal care utilization. Boston SPRANS Prenatal Care Project, Massachusetts Department of Public Health, 1987.

14 MC Donald TP, Cobrun AF. The Impact of variations AFDC and Medicaid eligibility in prenatal care utilization, Portland: Health Policy Unit, Human Services Development Institute, University Southern Maine, 1986.
15 Quelopana Del Valle, Ana María. Predictores del inicio del control prenatal en mujeres del área metropolitana de Monterrey. [Tesis de Doctorado]. México: Universidad Autónoma de Nuevo León, Facultad de enfermería, 2006:131.

16 Tamez S, Valle RI, Eibenschutz C, Méndez I. Adaptación del modelo de Andersen al contexto mexicano: acceso a la atención prenatal. Salud Pública de México, 2006;48(5):418-429.

17 Kébreau P, Crandall L, Saint-Jean G. Prenatal care utilization in rural areas and urban areas of Haiti. PAHO Journal 2005;18(2):84-92.

18 OMS. Diagnóstico cualitativo de la atención en salud sexual y reproductiva en Bolivia. Ginebra. 1996.

19 Villate, Yannet. Factores asociados a la no utilización de los servicios de control prenatal en el municipio de Duitama [tesis de maestría]. Colombia: Universidad del Valle, Facultad de salud. 2008. 68 p.

20 Vecino A. Determinants of demand for antenatal care in Colombia. Universidad del Rosario, facultad de economía, septiembre de 2007.

21 Valadez I., et al. Atención prenatal: Conocimientos, actitudes y cuidados alternos en Jalisco. En: Red de Revistas de América Latina y el Caribe, España y Portugal. Guadalajara-México, 2001; 3(1):39.

22 Palacio M. El estancamiento de la mortalidad materna: un problema evitable: Colombia. Bogotá: Organización Panamericana de la Salud, 2004. 


\title{
POSGRADOS CLÍNICOS
}

\section{ESPECIALIZACIÓN EN EPIDEMIOLOGÍA}

Registro ICFES No. 111456140404100113100

Creada mediante acuerdo Consejo Superior Universitario No. 009 del 10-05-1999

Registro calificado de calidad Res. No 3299 del 3-06-2008, Ministerio de Educación Nacional.

$\begin{array}{ll}\text { Denominación Académica: } & \text { Programa de Especialización en Epidemiología } \\ \text { Modalidad: } & \text { Semipresencial } \\ \text { Duración: } & 1 \text { año } \\ \text { Cupos: } & 30 \text { Anuales } \\ \text { Título: } & \text { Especialista en Epidemiología }\end{array}$

\section{ESPECIALIZACIÓN EN ENFERMERÍA NEFROLÓGICA Y UROLÓGICA}

Registro calificado MEN No. 9906 de Diciembre 23 de 2008.

\author{
Denominación Académica: Programa de Especialización en Enfermería Nefrológica y urológica \\ Modalidad: Presencial \\ Duración: 3 semestres \\ Cupos: 6 Anuales \\ Título: $\quad$ Especialista en Enfermería Nefrológica y urológica
}

\section{ESPECIALIZACIÓN EN ENFERMERÍA CUIDADO CRÍTICO}

Registro calificado MEN No. 9905 de Diciembre 23 de 2008.

\author{
Denominación Académica: Programa de Especialización en Enfermería Cuidado Crítico \\ Modalidad: \\ Presencial \\ Duración: \\ 3 semestres \\ Cupos: \\ 6 Anuales \\ Título: \\ Especialista en Enfermería Cuidado Crítico
}

